\title{
Structure-Based Drug-Drug Interaction Detection via Expressive Graph Convolutional Networks and Deep Sets (Student Abstract)
}

\author{
Mengying Sun, ${ }^{1}$ Fei Wang, ${ }^{2}$ Olivier Elemento, ${ }^{2}$ Jiayu Zhou ${ }^{1}$ \\ ${ }^{1}$ Michigan State University, ${ }^{2}$ Weill Cornell Medicine \\ sunmeng2@msu.edu, \{few2001, ole2001\}@med.cornell.edu, jiayuz@msu.edu
}

\begin{abstract}
In this work, we proposed a DDI detection method based on molecular structures using graph convolutional networks and deep sets. We proposed a more discriminative convolutional layer compared to conventional GCN and achieved permutation invariant prediction without losing the capability of capturing complicated interactions.
\end{abstract}

\section{Introduction}

Drug-drug interaction (DDI) occurs when two drugs are prescribed together, causing synergistic or antagonistic effects. Studies have reported that $30 \%$ of the adverse drug reactions are induced by DDI, among which some result in irreversible severe consequences. Therefore, identification of DDI is crucial for treatment effectiveness and patient safety. Computational methods have been proposed to detect DDI, including recent efforts that apply deep learning and graph convolutional networks (GCNs) that exploit non-Euclidean structures from raw structured inputs. However, the designs of most existing GCNs cannot fully capture the molecular structures and the interaction prediction often reduce to a simple similarity measure, therefore lead to suboptimal predictive performance. In this paper, we propose a GCN based framework for type-specific DDI identification from molecular structures. The proposed framework includes an encoder with more expressive GCN layers and a decoder that captures more complicated interactions while preserves permutation invariant on inputs. We evaluate our method in a real-world dataset and demonstrate improvements on each module as well as on the entire framework.

\section{Method}

The proposed framework is illustrated in Figure 1.

Graph Convolutional Encoder A graph convolutional layer encodes molecular structures by aggregating neighborhood atom features. The expressive power of a GCN refers to its ability to map two different neighborhood structures to different locations (Xu et al. 2018). In order to achieve

Copyright (c) 2020, Association for the Advancement of Artificial Intelligence (www.aaai.org). All rights reserved.

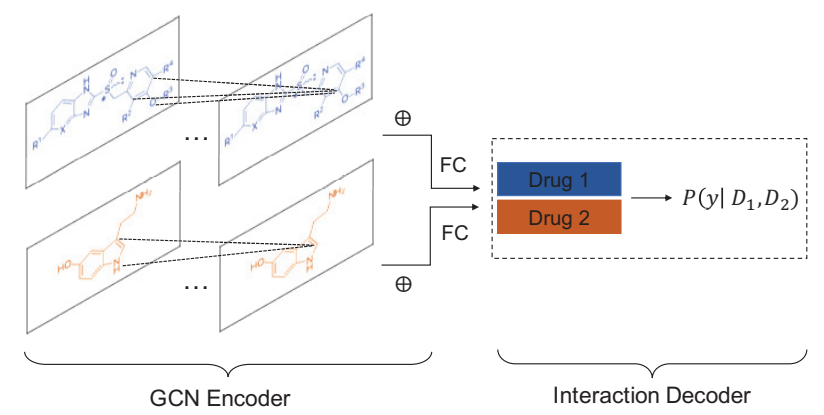

Figure 1: Illustration of the overall proposed framework. A pair of drugs are fed into the twin GCN branches for learning atom-wise features. The drug representation is then obtained by summing all atom features. A fully-connected layer (FC) followed by an interaction decoder outputs the likelihood that two drugs interact with each other.

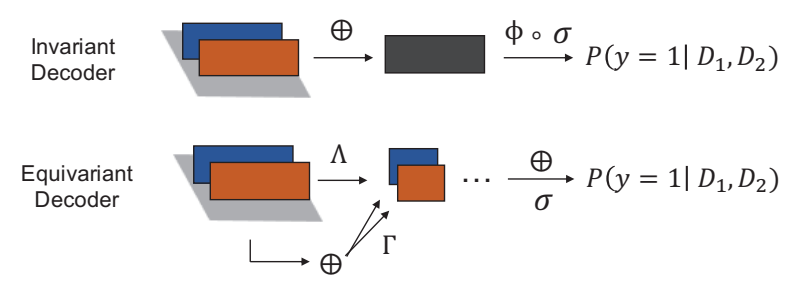

Figure 2: Illustration of interaction decoders (2 variants).

the maximum expressive power, MLPs instead of 1LP (single layer perceptron) before neighborhood aggregation is adopted in the proposed GCN layer ${ }^{1}$. Sum pooling is used as the aggregation function. The final representation of a drug is obtained by summing all atom representations.

Deep Sets Decoder The interaction between two drugs should be symmetric, i.e., the proposed model should output the same results regardless of permutation of the two drugs. Such symmetric design could potentially reduce model com-

\footnotetext{
${ }^{1} 1 \mathrm{LP}$ and MLP do NOT mean one and multiple graph convolutional layers, but rather the transformation used before neighborhood aggregation in a single convolution layer.
} 

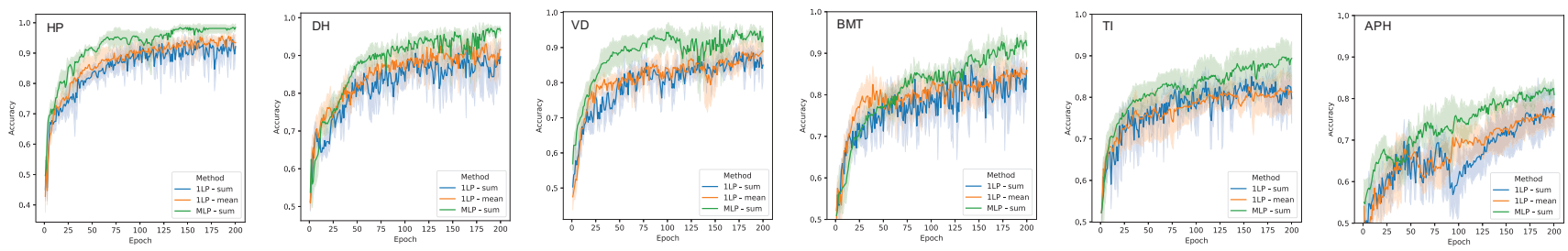

Figure 3: Convergence comparisons for different GCN settings for all tasks (better view in color, zoom if needed). For each DDI type, 5-fold training were conducted. Solid lines denote average accuracy while shadow denotes variation among 5 folds. Non-overlap shadow indicates significant difference of average performance (green: proposed GCN layer).

Table 1: 5-fold average generalization performance of different methods for the chosen DDI types (best performance highlighted bold. For equal values, standard deviation was further compared to determine the winner. Last 3 rows are proposed methods).

\begin{tabular}{c|cc|cc|cc|cc|ccc|c}
\hline DDI type & \multicolumn{2}{|c|}{ HL } & \multicolumn{2}{c|}{ DH } & \multicolumn{2}{c|}{ VD } & \multicolumn{2}{c|}{ BMT } & \multicolumn{3}{c|}{ TI } & \multicolumn{2}{c}{ AP } \\
\hline Method / Metrics & AUC & F1 & AUC & F1 & AUC & F1 & AUC & F1 & AUC & F1 & AUC & F1 \\
\hline Raw-DP & 0.763 & 0.714 & 0.719 & 0.678 & 0.792 & 0.733 & 0.777 & 0.714 & 0.789 & 0.694 & 0.599 & 0.622 \\
GCN-1LP-sum & 0.838 & 0.695 & 0.800 & 0.631 & 0.801 & 0.752 & 0.792 & 0.677 & 0.853 & 0.797 & 0.620 & 0.538 \\
GCN-1LP-mean & 0.837 & 0.699 & 0.820 & 0.653 & 0.822 & 0.767 & 0.839 & 0.737 & 0.863 & 0.802 & 0.647 & 0.614 \\
GCN-MLP-sum (EPGCN) & 0.875 & 0.711 & 0.879 & 0.830 & 0.864 & 0.798 & 0.843 & 0.796 & 0.901 & 0.815 & 0.678 & 0.617 \\
GATN-Deepset-1 & 0.841 & 0.804 & 0.858 & 0.845 & 0.857 & 0.858 & 0.869 & 0.845 & 0.885 & 0.869 & 0.675 & 0.652 \\
EPGCN-Deepset-1 & 0.907 & 0.850 & 0.922 & 0.857 & 0.903 & 0.831 & $\mathbf{0 . 9 1 2}$ & $\mathbf{0 . 8 4 9}$ & $\mathbf{0 . 9 2 8}$ & 0.849 & $\mathbf{0 . 7 2 7}$ & 0.668 \\
EPGCN-Deepset-2 & 0.902 & 0.857 & 0.930 & 0.869 & $\mathbf{0 . 9 0 7}$ & $\mathbf{0 . 8 4 0}$ & 0.889 & 0.815 & 0.917 & $\mathbf{0 . 8 5 2}$ & 0.727 & $\mathbf{0 . 6 8 1}$ \\
EPGCN-Deepset-3 & $\mathbf{0 . 9 2 6}$ & $\mathbf{0 . 8 7 8}$ & $\mathbf{0 . 9 4 0}$ & $\mathbf{0 . 8 9 8}$ & 0.895 & 0.823 & 0.884 & 0.823 & 0.901 & 0.843 & 0.686 & 0.667 \\
\hline
\end{tabular}

plexity but with the risk of failing to capture complicated interactions. Therefore, other than using conventional dot product, we consider modeling such interactions via deep sets (Zaheer et al. 2017). The proposed decoder has two variants. An invariant decoder adds up representations of objects in the set and then apply nonlinear transformations. An equivariant decoder models interaction via neural network layers with designed weights, and can be stacked multiple times in order to grow deep as shown in Figure 2. The entire model is optimized by minimizing the cross-entropy loss.

\section{Experiment}

The entire framework consists of a GCN encoder and an interaction decoder. To evaluate the potential benefits brought by each module and their combination, we consider following scenarios: 1) fix the decoder, evaluate the proposed encoder; 2) fix the encoder, evaluate the proposed decoder; 3) evaluate the entire framework. We conduct experiments on a DDI dataset (Zitnik, Agrawal, and Leskovec 2018) which contains 1317 unique adverse reactions caused by 63,473 unique drug pairs. Since the number of drug pairs varies among all DDI types, we randomly pick 6 DDI types which span the region of 1 st and 3rd quantile as the final tasks.

Results We compare the discriminative power of proposed and conventional GCN layer by examining the convergence and training accuracy for different tasks. The results are shown in Figure 3, suggesting that the proposed GCN has a larger discriminative power compared to other GCN variants. Given the same GCN encoder, performance of different decoders are shown in Figure 4. The generalization performance of the entire framework and baselines are in Table 1. Overall, the proposed GCN encoder in combination with deep sets decoders produces the best performance among all competing methods across all tasks.

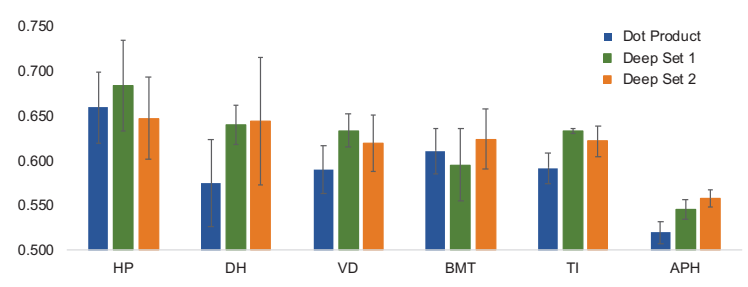

Figure 4: 5-fold average AUC for different decoders.

\section{Acknowledgments}

This material is based upon work supported by National Science Foundation under Grant IIS-1749940, IIS-1615597, and Office of Naval Research N00014-17-1-2265.

\section{References}

Xu, K.; Hu, W.; Leskovec, J.; and Jegelka, S. 2018. How powerful are graph neural networks? arXiv:1810.00826.

Zaheer, M.; Kottur, S.; Ravanbakhsh, S.; Poczos, B.; Salakhutdinov, R. R.; and Smola, A. J. 2017. Deep sets. In NIPS, 3391-3401.

Zitnik, M.; Agrawal, M.; and Leskovec, J. 2018. Modeling polypharmacy side effects with graph convolutional networks. arXiv:1802.00543. 